\title{
Analysis of agronomic traits of mungbean (Vigna radiata) accessions from the World Vegetable Gene Bank (Taiwan)
}

\author{
Alena Sokolkova \\ Peter the Great St. Petersburg \\ Polytechnic University, St. Petersburg, \\ Russia \\ alyonasok@yandex.ru
}

Margarita A. Vishnyakova

Federal Research Centre All-Russian

N.I. Vavilov Institute of Plant Genetic

Resources (VIR), St. Petersburg, Russia

m.vishnyakova@vir.nw.ru

Chau-Ti Ting

National Taiwan University, Taipei

106, Taiwan

ctting@ntu.edu.tw
Marina Burlyaeva

Federal Research Centre All-Russian

N.I. Vavilov Institute of Plant Genetic Resources (VIR), St. Petersburg, Russia m.burlyaeva@vir.nw.ru

Roland Schafleitner

World Vegetable Center, Shanhua, Tainan 74199, Taiwan

roland.schafleitner@worldveg.org

Sergey V. Nuzhdin

University of Southern California, Program in Molecular and

Computational Biology, Dornsife

College of Letters Arts \& Sciences, Los Angeles, CA 90089 USA

snuzhdin@usc.edu

Maria G. Samsonova

Peter the Great St. Petersburg

Polytechnic University, Department of

Applied Mathematics, St. Petersburg, Russia

m.g.samsonova@gmail.com
Eric von Wettberg

University of Vermont, Department of Plant and Soil Science, Burlington, VT 05405, USA

eric.bishop-von-wettberg@uvm.edu

Tatjana Valiannikova

Kuban Branch of Federal Research

Centre All-Russian N.I. Vavilov

Institute of Plant Genetic Resources

(VIR), Krasnodar region, Russia

fitolaka61@yandex.ru

Cheng-Ruei Lee

National Taiwan University, Taipei

106, Taiwan

chengrueilee@ntu.edu.tw

\begin{abstract}
The World Vegetable Gene Bank, in Taiwan, houses a unique genebank of mungbean. Here we analyze the genomes of 293 accessions that represent the major market classes. GWAS analysis conducted on phenotypic data obtained in Astrakhan experimental station of VIR in 2019 and multi-environmental GWAS analysis with phenotypic data obtained in different locations and time periods (Taiwan 1984, Taiwan 2018, India 2016, Kuban 2018, Astrakhan 2019) identified a large number of genome intervals and potential gene candidates that may affect important agronomic traits. To find out the effects of different climatic conditions on phenotypic traits, the data was subjected to AMMI and GGE biplot analyses. Phenotyping the mini-core collection of mungbean in different climatic locations can estimate phenology traits such as maturation. Uncovering SNPs for these traits will speed breeding efforts for production of mungbean suitable for cultivation in temperate regions.
\end{abstract}

Keywords - mungbean (Vigna radiata), GWAS analysis, candidate genes, AMMI, GGE biplot

\section{Motivation and aim}

\section{Motivation}

Vigna radiata is important Asian pulse crop, important as dried seeds, sprouts, and in the green form as vegetables. With good heat and drought tolerance, this crop is often grown as summer pulses in contrast to winter pulses such as chickpea and lentil, making it important for food security and nutritional diversity in many parts of Asia. The World Vegetable Gene Bank, in Taiwan, houses a unique genebank of mungbean [1]. Here we analyze the genomes of 293 accessions that represent the major market classes.
Aim

Earlier we have performed Genome-wide association study (GWAS) to find associations between accessions of the mini-core collection and phenotypic data obtained in Kuban experimental station of VIR in 2018 under stress weather conditions. GWAS analysis identified a block of four SNPs within the region of strong linkage associated with maturation in stress weather conditions in which genes encoding zinc finger A20 and AN1 domain stressassociated protein are located. SAPs are known to prevent the loss of yield caused by environmental stresses [2]. In 2019 the phenotypic data was obtained in Astrakhan experimental station of VIR. GWAS analysis was performed with phenotypic data obtained in 2019. Previously, the mini-core collection has been grown in common field plots in several locations, including Taiwan (1984, 2018), and India in 2016. Multi-Environmental GWAS analysis was performed with phenotypic data obtained in different locations and time periods and AMMI (Additive Main effects and Multiplicative Interaction) and GGE (Genotype plus Genotype-Environment interaction) biplot analyses were performed to determine the effect of genotype, environment and their interaction for phenotypic traits measured under different climatic conditions.

\section{Methods}

Genotyping by sequencing (GBS) of the mini-core collection's 293 mungbean accessions from different countries identified 8,466 segregating single nucleotide polymorphisms (SNP). For these SNP calls we implemented inclusion criteria: minor allele frequency (MAF) more than 3\%, genotype call-rate more than $90 \%$. 5,041 SNPs passed all filters and remained for further analysis. We performed GWAS to find associations 
between mungbean accessions and phenotypic data obtained in Astrakhan experimental station of VIR in 2019 and multi-environmental GWAS analysis with phenotypic data obtained in different locations and time periods (Taiwan 1984, Taiwan 2018, India 2016, Kuban 2018, Astrakhan 2019). To find out the effects of different climatic conditions on phenotypic traits, the data was subjected to AMMI and GGE biplot analyses.

\section{Results}

GWAS analysis conducted on phenotypic data obtained in Astrakhan experimental station of VIR in 2019 and multienvironmental GWAS analysis with phenotypic data obtained in different locations and time periods (Taiwan 1984, Taiwan 2018, India 2016, Kuban 2018, Astrakhan 2019) identified a large number of genome intervals and potential gene candidates that may affect important agronomic traits. The results indicated that AMMI and GGE biplot analyses are informative methods to evaluate the effects of different climatic conditions on phenotypic traits. The mini-core collection of mungbean established by the World Vegetable Center is a valuable resource for mungbean breeding. Phenotyping of this collection in different climatic locations can estimate phenology traits such as maturation. Uncovering SNPs for these traits will speed breeding efforts for production of mungbean suitable for cultivation in temperate regions.

\section{ACKNOWLEDGMENT}

Supported by the RSCF (18-46-08001).

\section{REFERENCES}

[1] Schafleitner R et al. (2015) The AVRDC - The World Vegetable Center mungbean (Vigna radiata) core and mini core collections. BMC Genomics. 16(1):344. doi: 10.1186/s12864-015-1556-7.

[2] Ben Saad R et al. (2019) Functional domain analysis of LmSAP protein reveals the crucial role of the zinc-finger A20 domain in abiotic stress tolerance. Protoplasma. 256(5):1333-1344. doi: 10.1007/s00709-019-01390-2. 